\title{
Risk Identification of Safety Management Capability in Coal Mine Enterprise and its Relationship Model
}

\author{
He Yerong ${ }^{*}, 1,2$, Li Huizong ${ }^{3}$ and Wang Xiangqian ${ }^{3}$ \\ ${ }^{1}$ School of Mining and Safety Engineering, Anhui University of Science \& Technology, Huainan, Anhui, 232001, P.R. \\ China \\ ${ }^{2}$ School of Economic and Management, Huainan Normal University, Huainan, Anhui, 232001, P.R. China \\ ${ }^{3}$ School of Economic and Management, Anhui University of Science \& Technology, Huainan, Anhui, 232001, P.R. China
}

\begin{abstract}
According to the connotation of the coal mine enterprise's safety management capability, we first utilize the method of quantitative analysis to assess the risk affecting factors of the coal mine enterprise's safety management capability, then, the main capability factors including 5 hierarchies and 22 items are identified, the 5 hierarchies are safety administrative management ability, safety behavior management ability, safety technology management ability, safety information management ability, and safety innovation management ability. Based on this, the structural equation model of the coal mine safety management capability factors is constructed, and the paper discloses the comprehensive influence of these risk factors to the coal mine enterprise's safety management capability, and we also discover the specific influence paths. The research can provide decisions on the coal mine enterprise's essential safety.
\end{abstract}

Keywords: Coal mine enterprise, risk factor, relationship model, safety management capability.

\section{INTRODUCTION}

China is rich in coal resources. The output of coal in China has ranked first since the year of 1990, and the totality of coal output in 2013 has exceeded 3.5 billion tons. Although coal consumption has retarded recently, coal industry dominates in primary energy production and consumption at present in China, which is of great significance in accelerating social and economic development [1] Experts estimate that coal consumption will still account for about $50 \%$ of Chinese energy consumption by the year 2050. As a result, coal industry will remain dominant in Chinese energy industry in the long run.

Problems of security in coal production and management keep severe because of complex coal occurrence in China, natural disasters, safety management to be not in place, coal mine employees' poor sense of safety and knowledge and skill, and illegal mining in some privately operated coal mines. According to the statistics data in State Administration of work safety, China has largely strengthened the force to monitor safety management in coal mine so that the rate of coal mine accidents has dropped relatively with the fatality in coal mine accidents diminishing year by year (As shown in Fig. 1). While in contrast with the occidental countries gross of coal mine accidents is still higher [2]. Overall analysis and research of Data in Fig. (1) combining with Coal mine Accidents in China and expert review reveals that $90 \%$ of coal mine accidents results from human factors [3]. Human un-safety factors are caused by safety management substantially, and by poor capability of safety management objectively. A large number of researches [4-6] indicate efficient safety management capability will make effective decrease in occurrence of coal mine accidents, lower the degree of the loss by accidents, therefore, efficiency of safety management will be increased.

Government and relevant departments have increasing given priority to couples of significant issues. For examples, how to identify risk factors impacting safety management capability in coalmine enterprises, how to clarify relations among these factors in order to promote safety management capability and to guarantee realization of essential safety in coal mine enterprises. According to results of previous investigations and researches [7, 23], based on objectives of safety management capability in coal mine enterprises, characteristic of coal mine accidents were used to analyze these risk factors, and relationship model of these factors was established, then the model was tested, empirical study is made finally. The paper discussed all kinds of risk factors and relations among them, the quality of factors' influence path and influence degree of safety management capability. Accordingly, a novel perspective from safety management capability will be provided for preventing coal mine accidents.

\section{STUDY OF SAMPLES SELECTION}

Safety management capability in coal mine enterprises refers to the management capability preformed concerning how to avoid or reduce the probability of coal mine accidents and lower the loss and damage caused by accidents. Risk factors impacting safety management capability differ 


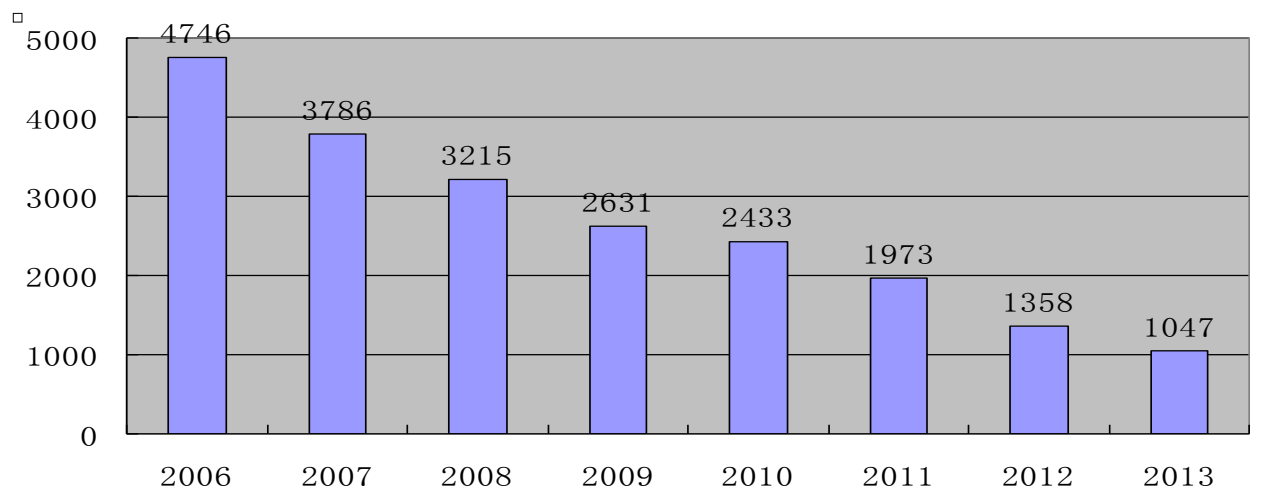

Fig. (1). Death tolls of coal mine accidents on 2006-2013.

variously. Based on four layers such as staff, team, equipment, surroundings and institutions, structure equation was applied in Liu and Li's studies [8] to analyze these risk factors. From the viewpoint of strategic orientation, Qi and Liu [9] investigated components of safety management capability, and they built an evaluation model of this capability on a basis of institutions, staff, external and internal environment. Li [10] established SEM model of safety management capability from staff, equipment, surroundings and institutions, and they used the SEM to research components of the capability and relations among the components. Having studied management faults behavior in major accidents in Chinese coal mines, Cheng [11] hold managers' demographic characteristics, organizational commitment and characteristics, self-efficiency, and production conditions are of significance in major coal mine accidents. Indeed the management faults reflect objectively inadequacy of safety behavior management capability.

According to research results home and broad, the paper selected five coal colleges and universities, 12 coal mines (five of which are privately operated coal mines) in Huainan and Huaibei as research objects, issued 360 questionnaires in all, and retrieved 336 questionnaires (with 16 ones invalid) which accounts for $68.5 \%$ of all the questionnaires, and the questionnaires valid accounts for $84.9 \%$. Then software SPSS18.0 was used to analyze the reliability and validity of the data gained in the investigation. Generally, reliability is

Table 1. Values of cronbach's alpha and CITC of coal mine safety management capability risk factors.

\begin{tabular}{|c|c|c|c|c|c|}
\hline Item & $\begin{array}{l}\text { Cronbach's } \\
\text { Alpha }\end{array}$ & CITC & Item & $\begin{array}{l}\text { Cronbach's } \\
\text { Alpha }\end{array}$ & CITC \\
\hline Organizational safety behavior & 0.713 & 0.546 & safety in work conditions & 0.706 & 0.536 \\
\hline $\begin{array}{l}\text { organization individual safety } \\
\text { behavior }\end{array}$ & 0.808 & 0.513 & Project quality conditions & 0.721 & 0.541 \\
\hline Organizational culture & 0.411 & 0.214 & $\begin{array}{l}\text { Construction of safety behavior } \\
\text { incentive mechanism }\end{array}$ & 0.715 & 0.512 \\
\hline management and communication & 0.736 & 0.521 & $\begin{array}{l}\text { Construction of safety management } \\
\text { information system }\end{array}$ & 0.751 & 0.532 \\
\hline $\begin{array}{c}\text { Safety management quality of } \\
\text { executive level }\end{array}$ & 0.702 & 0.510 & $\begin{array}{l}\text { Information gathering and } \\
\text { managing }\end{array}$ & 0.710 & 0.510 \\
\hline $\begin{array}{c}\text { Construction of safety management } \\
\text { systems }\end{array}$ & 0.714 & 0.515 & Information source and quality & 0.721 & 0.501 \\
\hline $\begin{array}{c}\text { Executives' and employees' degree } \\
\text { of education }\end{array}$ & 0.321 & 0.201 & Effect of using information & 0.704 & 0.521 \\
\hline $\begin{array}{l}\text { ability of organization and } \\
\text { coordination }\end{array}$ & 0.806 & 0.512 & $\begin{array}{c}\text { Administration execution of safety } \\
\text { management }\end{array}$ & 0.707 & 0.506 \\
\hline Improvement degree of law system & 0.721 & 0.513 & $\begin{array}{l}\text { Adaptation of safety management } \\
\text { ability }\end{array}$ & 0.714 & 0.513 \\
\hline Vocational skills level & 0.715 & 0.514 & $\begin{array}{c}\text { Decision-making power in safety } \\
\text { and risk }\end{array}$ & 0.736 & 0.514 \\
\hline Reliability of equipment & 0.711 & 0.521 & $\begin{array}{c}\text { Foresight of safety management } \\
\text { idea }\end{array}$ & 0.740 & 0.521 \\
\hline \multirow[t]{2}{*}{$\begin{array}{l}\text { Safety resource integration } \\
\text { competence }\end{array}$} & 0.710 & 0.501 & $\begin{array}{l}\text { analysis and judgment ability of } \\
\text { safety issues }\end{array}$ & 0.724 & 0.510 \\
\hline & & & totality & 0.913 & 0.546 \\
\hline
\end{tabular}


Table 2. KMO and Bartlett tests of coal mine safety management capability risk factors.

\begin{tabular}{|l||c|c|}
\hline \multirow{2}{*}{ Kaiser-Meyer-Olkin } & Measure of Sampling Adequacy & 0.834 \\
\cline { 2 - 3 } & Approx. Chi-Square & 1548.32 \\
\hline \multirow{2}{*}{ Bartlett's Test of Sphericity } & df & 300 \\
\cline { 2 - 3 } & Sig. & .000 \\
\hline
\end{tabular}

tested by Cronbach's Alpha value which should be over 0.7 [12] and CITC value which should be not less than 0.3 . In fact, the CITC value is more available when it is more than 0.5 . And validity is tested by KMO and Bartlett sphericity tests. The test results of reliability are shown in Table $\mathbf{1}$ and test results of validity are shown in Table 2 .

As mentioned in Table 1, the value of Cronbach's Alpha is 0.913 on average. Because organization culture and employees' degree of education failed to be tested, the two items were therefore deleted. The number of all the items is left 22. The average $\alpha$ value of each single factor is larger than standard values 0.7 , while the CITC value is larger than 0.5 on averages. It was concluded that the questionnaire is of higher credibility. As the Table 2 shows, when KMO is larger than 0.9 for which factor analysis is quite advisably made; when KMO is between 0.8 and 0.9 factor analysis is advisably made; between 0.7 and 0.8 , the effect of factor analysis is ok; while when $\mathrm{KMO}$ is 0.6 , the effect is very poor; but when it is below 0.5 , factor analysis is inadequate to make. The KMO value in this paper is 0.834 , factor analysis is quite advisably made accordingly. The value of Bartlett's Test of Sphericity in the paper is 1548.32 with the relevant probability Sig 0.000 , which indicates that correlation matrix differs remarkably from unit matrix. And the variables are fit for factor analysis. As a result, the questionnaire is of relatively higher credibility and validity.

\section{STUDY OF VARIABLE DESIGN}

Safety management capability in coal mine is the essential element to realize this management in coal mine, the quality of Safety management capability decides the effect of the capability. The coal mine enterprises survive and grow in a dynamic situation, requiring enterprises to have better management system and efficient administrative execution. As an important factor in safety management capability in coal mine, safety administrative management capability warranties highly efficient organization operation and person's code of behavior. Besides, information is the basis of safety management decision in coal mine, promptness of collecting information, source of information and information quality are immediately decisive in validity of safety management decision. Time-dependent nature of Safety management capability in coal mine demands innovative safety management capability. Coal mine safety is a dynamic system composed of person, machine, surroundings and information, demanding the capability of resources integration. On the other hand, coal mine safety management is based on safety of machines, equipment and surrounding. At the same time, safety technology management significantly guarantees the safety of machines and equipment, surroundings and person. Factors impacting safety management capability and their interrelation can be identified so as to offer theoretical reference for studying coal mine safety management capability at present.

In this paper SPSS18.0 was used to analyze the 22 risk factors impacting coal mine safety management capability so that major factors impacting coal mine safety management capability can be extracted. Then by rotating factors there are five factors with whose characteristic root is larger than 1 , this explained $67.85 \%$ information of all variables, namely, 22 variables can be effectively classified into five types as shown in Table 3.

Safety management behavior management capability means how to encourage and restrain organizational and individual safety behavior, the capability contains organization safety behavior management, individual safety behavior management, incentive mechanism construction and leadership and communication. While safety management administrative management capability covers construction of how to examine safety management system, and of improvement degree of relevant law system. Moreover, safety administrative executive capability consists of improvement degree of relevant law system, management system, industry norm, administrative executive capability, and organization and coordination ability as well.

Safety technology management ability means the ability to guarantee the safety of technology, equipment and surroundings. It contains safety technology management, equipment management, project quality management and surroundings safety guarantee. While safety information management ability refers to construction of safety management information system, collection and organization of safety information, information source and information quality guarantee, and effect feedback of information utilization. At the same time, safety innovation management ability covers adaptation of safety management ability, foresight of safety management thinking, judgment and analysis of safety issues, decision power of safety risk and ability of safety source integration.

\section{STUDY HYPOTHESIS}

Coal mine safety management capability is composed of a few comprehensive capacities. As the above mentioned, repeated group discussions encourage the paper to propose the following hypotheses.

H1: Safety behavior management level impacts coal mine safety management capability noticeably. The more standard the organization and individual behaviors become, the more beneficial they are to safety behavior management. The better incentive mechanism is built, the better management and communication are, the more powerful safety management capability will become. Conversely, the 
bigger safety management risk become, the more likely the risk happens.

H2: Safety administration management level impacts coal mine safety management capability remarkably. The more strongly the management is aware of safety, the higher safety management quality becomes, the more beneficial they are to safety administration, and the less likely safety management capability is at risk; the better safety management system is built, the stronger safety administration execution capability is, the more powerful safety organization and coordination ability are becoming, the more law and institutional system is improved, the more beneficial they are to safety management. On the contrary, the bigger safety management risk become, the more likely the risk happens.

H3: Safety technology management level poses the outstanding impacts on coal mine safety management capability. The higher the employee's vocational skills are, the better the safety performance of equipment is, the higher the surrounding safety is, and the more beneficial they are to safety management. Contrarily, the bigger safety management risk become, the more likely the risk happens.

H4: Safety information management level impacts strongly on coal mine safety management capability. The better the safety management information system is constructed, the more beneficial it is to safety management; the more effectively information is collected and organized, the reliable information source is becoming, the higher information quality is, the better information is used, the beneficial they are to safety management. Contrarily, the bigger safety management risk become, the more likely the risk happens.

H5: Safety innovation management level influences coal mine safety management capability obviously. The stronger the adaptation of safety management capability becomes, the further foresight the management thinking is of, the higher safety innovation management level grows, the more beneficial they are to safety management. On the other hand,

Table 3. Coal mine safety management ability risk factors.

\begin{tabular}{|c|c|c|c|}
\hline Level-One Variable & Level-Two Variable & Observational Variable & Symbol \\
\hline & \multirow{4}{*}{ (SBMA) } & Safety behavior of organization and individual & $V_{1}$ \\
\hline & & Organizational safety culture construction & $V_{2}$ \\
\hline & & Incentive mechanism construction & $V_{3}$ \\
\hline & & Ianagement and communication & $V_{4}$ \\
\hline & \multirow{5}{*}{ (SAMA) } & Safety management quality of executive level & $V_{5}$ \\
\hline & & Construction of safety management systems & $V_{6}$ \\
\hline & & executive ability of safety administration & $V_{7}$ \\
\hline & & ability of organization and coordination & $V_{8}$ \\
\hline & & Improvement degree of law system & $V_{9}$ \\
\hline & \multirow{4}{*}{ (STMA) } & Vocational skills level & $V_{10}$ \\
\hline & & Reliability of equipment & $V_{11}$ \\
\hline & & Project quality conditions & $V_{12}$ \\
\hline & & safety in work conditions & $V_{13}$ \\
\hline & \multirow{4}{*}{ (SIMA) } & Construction of information system & $V_{14}$ \\
\hline & & Information gathering and managing & $V_{15}$ \\
\hline & & Source and quality of information & $V_{16}$ \\
\hline & & effect of using information & $V_{17}$ \\
\hline & \multirow{5}{*}{ (SCMA) } & Adaptation of management ability & $V_{18}$ \\
\hline & & Foresight of management idea & $V_{19}$ \\
\hline & & Decision-making power in safety and risk & $V_{20}$ \\
\hline & & analysis and judgment ability of safety issues & $V_{21}$ \\
\hline & & Safety resource integration competence & $V_{22}$ \\
\hline
\end{tabular}


the more powerful the surroundings emergency of safety management capability becomes, the stronger the judgment and analysis ability of safety issues becomes, and the more beneficial they are to safety management. Contrarily, the bigger safety management risk become, the more likely the risk happens.

H6: Factors like safety behavior management capability, safety administration management ability, safety technology management ability, safety information management ability and safety innovation management ability play the big role in the high-order factor of coal mine safety management capability. In addition, these factors are of significant positive correlation.

\section{HYPOTHESIS AND TEST MODEL}

\subsection{Model Construction}

The concepts of coal mine safety management capability are too abstract to analyze precisely, and relations among variables remain complicated and immeasurable. As a result, the features of study objective and study subject decide structural equation as study tool. Structural Equation Model (SEM), proposed by Swedish statistician Karl G. Joreskog and his partner Dag Sorbom in 1960s-1970s, contains Measurement Model, structural equation. Measurement Model mainly means relation between latent variable and observation variable, namely, the relation between exogenous latent variable and exogenous observation variable; the relation between endogenous latent variable and endogenous observation variable. While, Structural Model mainly refers to relations among latent variables, this relation is divided into impact relation of exogenous latent variable on endogenous latent variable and mutual impact relations among endogenous latent variables [13]. As the major tool of modern social science and behavioral science, SEM can be used to analyze relation of complex factors, and to refine risk factors, and assess to optimization of index system. Nowadays, it has found its wide use in every field of social science and behavioral science [14-18].

The model in this paper covers the five exogenous latent variables such as management capability risk factor, safety administration management ability risk factor, safety technology management ability risk factor, safety information management ability risk factor and safety innovation management risk factor as well. These five exogenous latent variables contain four, five, four, four, five observation variables respectively, which conform to requirement of building structural equation. Moreover, the model in the paper mainly investigates the effect of all risk factors on coal mine safety management capability and interaction relations among these factors. Based on covariance matrix of the questionnaire made in the study, the software STATA12.0 is used to build the model. Operation results and model of structural equation of all variables are shown in Fig. (2).

Structural equation relies on theoretical inference and hypothesis, and reasonability of model construction. It also needs to be tested in virtue of relations between latent variable and observation variable, relations among latent variables. Then, after regulating measurement system of impact factors, SEM, MLE and software STATA12.0 are used to test the model. This model contains five risk factors of premise variables and one outcome variable. The premise variables are safety behavior management ability, safety administration management ability, safety technology management ability, safety information management, safety innovation management and safety management ability. The one outcome variable is safety management ability. The premise variable impacts the outcome variable comprehensively.

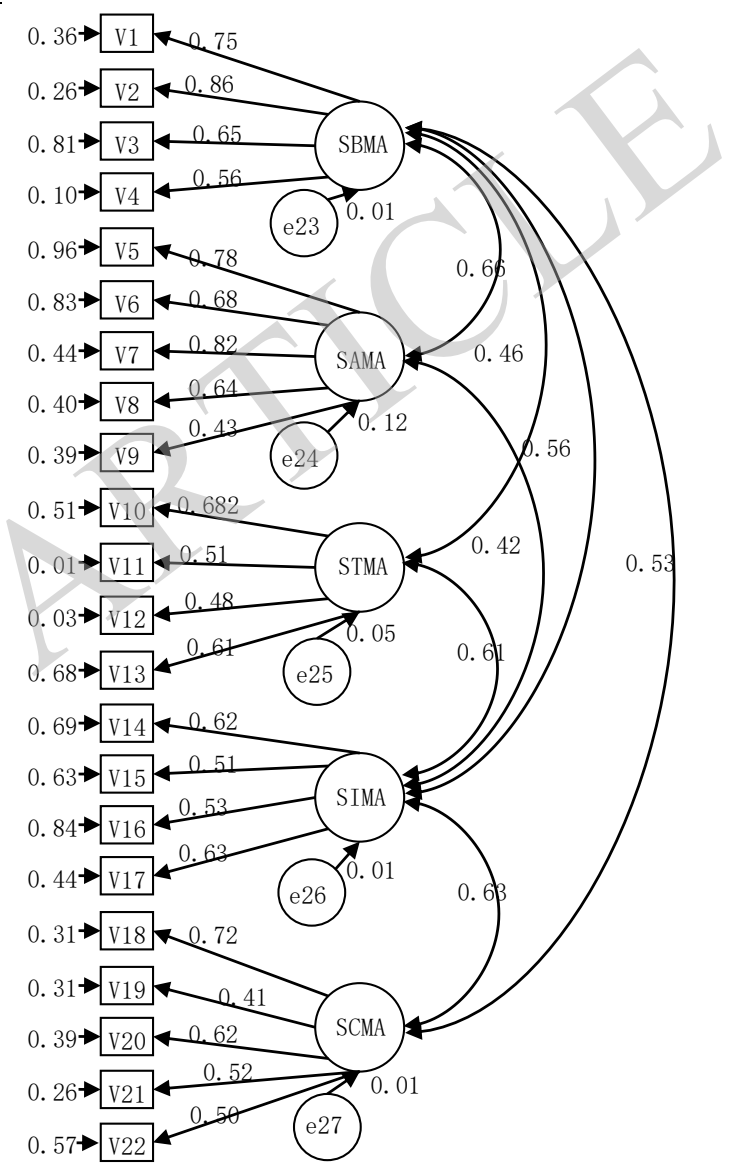

Fig. (2). Structural equation model of relationship in each factor and its computing results.

\subsection{Test and Correction of the Model}

Every link in the model was modified according to correction proposals given by the model, and parameters were tested again until the parameters of the model satisfied the demand. Parameter after correction is shown in Table 4 and the model after correction is shown in Fig. (3).

In general, the $\chi^{2} / d f$ value is between 2 and 5. The value in the paper is 3.17 (As shown in Fig. 3), which means the model is receivable. RMSEA (root-mean-square error) is usually below 0.1 . That is, the smaller the value of RMSEA, the better the model fitting is. When the value is below 0.05 the model fitting is first fate. While it is below 0.01 , the fitting is best $[19,20]$. 
The value of RMSEA in the paper is 0.0805 , meaning the fitting is very best. Besides, NNFI (non-normalized fit index) is between 0 and 1. NFI (normalized fit index) is also between 0 and 1 , the closer the value is to 1 , the better the fitting effect. When NFI $=1$, the fitting is best; but when $\mathrm{NFI}=0$, the fitting is worst. In the paper, NFI $=0.864$, $\mathrm{NNFI}=0.918$, which indicates the fitting is best [21]. CFI (Comparative fit index) means the degree of Comparative fit between the model to test and the model whose variables are entirely controlled. When CFI value is larger than 0.9 , it means the model is receivable. This value in the paper is 0.907 [22], which shows that the model fitting is much better.

GFI (Goodness Fit Index) and AGFI (Adjusted Goodness Fit Index) are used to reflect absolute fitness of the model. When the difference is smaller, the non-significant paths possibly contained in the model are less. Generally, when GFI and AGFI are larger than 0.9 respectively, the model fitting is better. If the index is higher, the model fitting is better. GFI and AGFI in the model after correction are 0.902, 0.913 respectively. The model has met GFI standard. Therefore, the whole fitting of the model is better. The model fitting obtained through operation in SEM established in the study show that the model can explain the data highly, that the discrepancy between the model and the data remains smaller, and that effectiveness of the model was verified better. The result of using the software STATA12.0 to test the model finds out that the model fitting is much better.

\subsection{SEM Path Analysis of SEM}

As shown above, for H1, safety behavior management posed the positive impact on coal mine safety management capability. The path coefficient is $0.875, \mathrm{H} 1$ holds water. At the same time, the secondary variables below H1 were verified, their path coefficients were $0.753,0.863,0.654$, 0.568 respectively, and they are larger than 0.5 , of which organization safety management behavior impact is obvious. While for $\mathrm{H} 2$, safety administration management plays remarkable role in coal mine safety management capability, its path coefficient is 0.758 , then $\mathrm{H} 2$ is true. And $80 \%$ of the secondary variables below $\mathrm{H} 2$ were verified. The first four path coefficients were $0.785,0.683,0.826$, and 0.645 , which were larger than 0.5 , of which safety management administrative execution had the largest path coefficient 0.826 . But the fifth path coefficient is 0.436 less than 0.5 , it failed to test. For H3, the path coefficient of safety technology management level was 0.614 which shows that $\mathrm{H} 3$ is basically true. The secondary variable hypothesis was partly verified with the third path coefficient 0.483 , which failed to test. But the other three variables left were larger than 0.5 , and they were verified. For H4, safety information management level with its path coefficient 0.603 impacted coal mine safety management capability noticeably, $\mathrm{H} 4$ was tested true. The secondary variable hypotheses below $\mathrm{H} 4$ were fundamentally tested, their path coefficients were larger than 0.5 . For H5, safety innovation management level with its path coefficient 0.721 impacted coal mine safety management capability dramatically. H5 was tested true because parts of the secondary variable hypotheses were tested true. Except the second path coefficient was 0.418 less than 0.5 , the other four path coefficients of the secondary variables were larger than 0.5 , they were tested true. Finally, for H6, impact path coefficients of safety behavior management, safety administration management, safety technology management, safety information management, and safety innovation management on coal mine safety management capability were $0.875,0.758,0.6147,0.603$, and 0.721 respectively. They all were larger than 0.5 with positive relation with safety management ability, showing that the key factors of five layers of coal mine safety management capability can be integrated into one factor and H6 holds water.

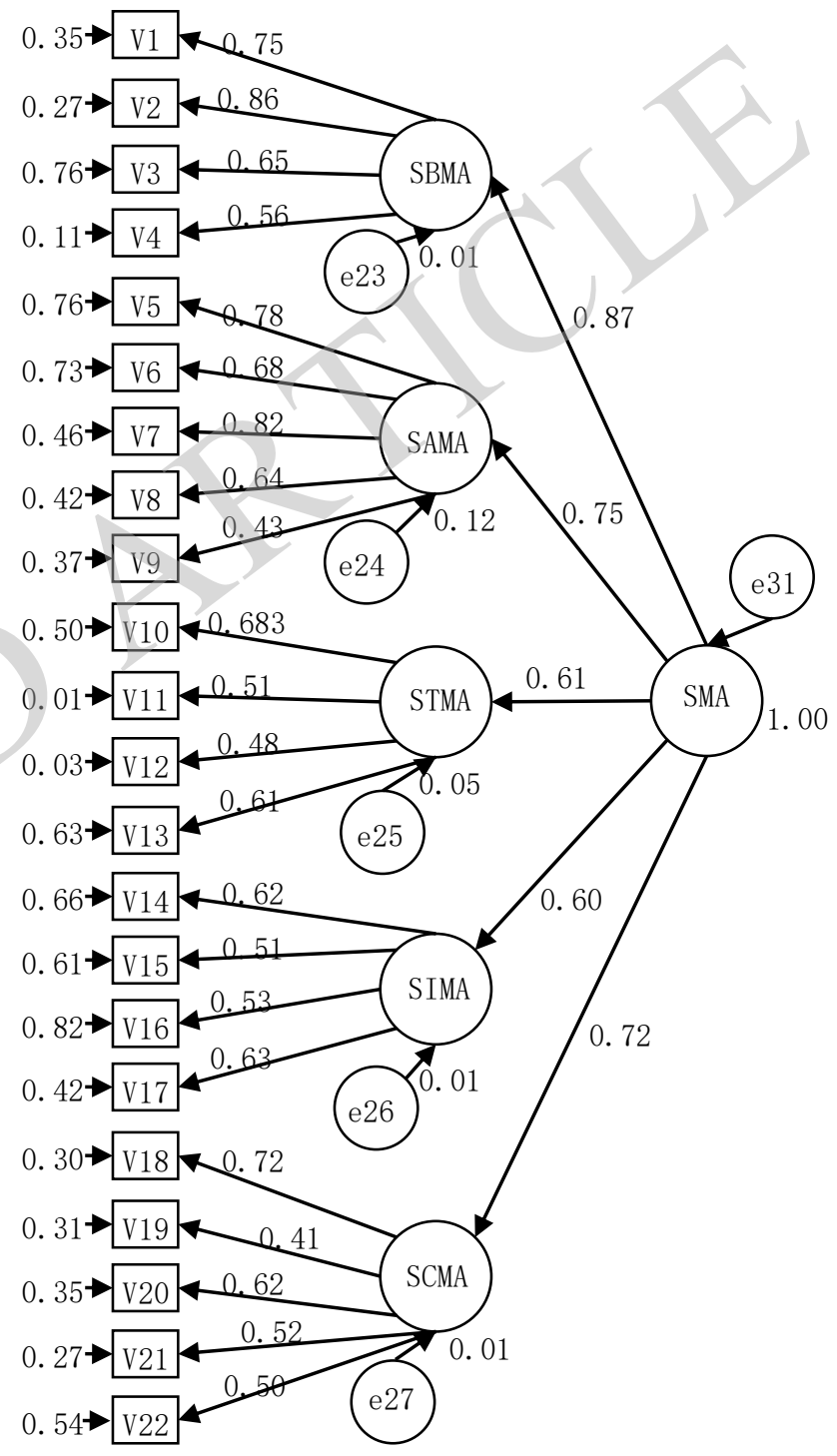

Fig. (3). Second-order factor structural equation model and risk pathways of coal mine safety management capability.

\section{CONCLUSION}

As standardized regression coefficient in SEM, path coefficient works as a standard to measure impact degree among all variables. Normalization processing of standardized index in Fig (3) found that weights of impact factors in safety behavior management in the secondary impact factors were $0.267,0.301,0.230$, and 0.202 respectively. And weights of impact factors in safety 
Table 4. Fitting results of coal mine safety management capability risk factor model.

\begin{tabular}{|c|c|c|c|c|c|c|c|c|}
\hline Chi-Square & DF & P & RMSEA & NFI & NNFI & CFI & GFI & AGFI \\
\hline \hline 856.39 & 270.86 & 0.00 & 0.0805 & 0.864 & 0.918 & 0.907 & 0.902 & 0.913 \\
\hline
\end{tabular}

administration management were $0.233,0.202,0.245,0.191$, and 0.059 respectively. Weights of impact factors in safety information management were $0.272,0.224,0.231$, and 0.273 respectively. Weights of impact factors in safety innovation management were $0.250,0.179,0.216,0.181$, and 0.174 respectively. The standardized index impacting coal mine safety management capability can be synthesized as the following index: $0.875,0.758,0.587,0.603,0.621$, and their comprehensive weights were $0.254,0.220,0.170,0.175$, and 0.181 respectively.

Through the analysis mentioned above, safety behavior management poses the largest impact on safety management capability during the process of coal mine safety management. For this reason, coal mine safety behavior management should be strengthened in order to decrease coal accidents. Safety organization behavior should be normalized so that law and regulations should be stipulated to teach employees in coal mine to build their sense of safety awareness and safety responsibility. On the other hand, the relevant mechanism of incentive and constraint should be established to encourage and control employees' behavior. Immediate communication with employees must be made so as to shorten the distance between the management and workers at the production line. By doing so, safety information can be kept unimpeded. At the same time, it is essential to enhance institutional construction, to improve administrative execution in the process of coal mine safety management. Moreover, safety technology is guarantee for coal mine safety; safety information is fundamental, so it is significant warranty to realize intrinsic safety in coal mine by constantly lifting coal mine safety technology level, enforcing safety information construction. Safety innovation management as the mainline runs through the whole safety management. As a result, division heads of coal mine safety management department are supposed to look far ahead and aim high, to plan as a whole, and to truly realize safety management concept innovation and safety management process innovation. They are in a good position to remove possible problems in the bud. Therefore, coal mine safety management can be implemented high efficiently.

\section{CONFLICT OF INTEREST}

The authors confirm that this article content has no conflict of interest.

\section{ACKNOWLEDGEMENTS}

This work was financially Supported by the National Natural Science Foundation of China (51374114); the National Natural Science Foundation of China (51474007); the Ministry of Education Humanities and Social Science Research on Youth Foundation of China (13YJCZH077); the Major Project Supported by College Humanities and Social Science of Anhui Province, China (SK2014ZD046); the
Planning Projects of Philosophies and Social Sciences Issued by Anhui Province, China (AHSK11-12D293).

\section{REFERENCES}

[1] G. Liu, and H. L. Song, "Research of China energy gap", China Mining Magazine, vol. 17, no.8, pp. 1-4, 2010.

[2] Z. Q. Song, "Development situation and direction of coal mining technology in China", The safety construction and risk management of important underground engineering, In: Proceedings of International Engineering Science and Technology Development Strategy High-End Forum, Beijing, China, 2012, pp. 178-188.

[3] S. C. Tian, and H. X. Li, "Probe into the frequency of coal mine accidents based on the theory of three types of hazards", China Safety Science Journal, vol. 17, no. 1, pp. 10-15, 2007.

[4] T. Z. Liu, "Evaluating of Chinese Firms Safety Management Capability", Journal of Beijing Institute of Technology (English Edition), vol.16 (SUPPL), pp. 2024-2026, 2007.

[5] H. J. Li, and T. Z. Liu, "Study on theory framework of safety management capability in coal mine", Journal of Safety Science and Technology, vol. 6, no. 5, pp. 143-146, 2010.

[6] C. $\mathrm{Xi}$, "Study on Construction mode of mine emergency management system", China Safety Science Journal, vol. 20, no. 1, pp. 159-164, 2010.

C. L. Lu, "Study on the Risk Identification, Risk Assessment and Risk Control of the Coal Enterprise", Master's Thesis, University of Xi' an Science and Technology, 2010.

[8] T. Z. Liu, and Z. X. Li, "Affecting factors of safety management capability about coal mine based on structural equation model", Journal of China Coal Society, vol. 33, no. 12, pp. 1452-1456, 2008.

[9] H. L. Qi, "Research on safety management capability of coal enterprises of strategic orientation", Coal Technology, vol. 32, no. 5, pp. 3-5, 2013.

[10] H. J. Li, "SEM modeling about the elements of safety management capability of coal mine", Safety in Coal Mines, vol. 2, pp. 145-148, 2011

[11] H. Chen, "The structural model of affecting factors of management misconduct in coal mine fatal accidents in China", Journal of China Coal Society, vol. 31, no. 5, pp. 689-696, 2006.

[12] R. F. Devellis, Scale Development: Theory and Application (Applied Social Research Methods), Sage Publication: California, 1991.

[13] J. T. Hou, Structural Equation Model and its Application. Educational Science Publishing House, Beijing, 2008.

[14] Y. F. Den, "Study on city emergency capability assessment system", Journal of Safety Science and Technology, vol. 12, pp. 3336, 2005.

[15] B. Paul, "Structural equation mode ling: adjudging model fit", Personality and Individual Differences, vol. 42, no. 5, pp. 815-824, 2007.

[16] T. J. Zhang, "The Positivistic Research on Correlation Between Knowledge Transferring and Formation of Core Competence in Chinese Enterprises", Doctor's Thesis, University of China Mining \& Technology, 2008.

[17] S. G. Li, "Structural equation model for influential factors of coal mine emergency rescue capability", Journal of Xi'an University of Science and Technology, vol. 32, no.1, pp. 8-12, 2012.

[18] Y. Shi, "Evaluation and application of Coal Mine's Emergency Rescue Capabilities Based on SEM Model", Master's Thesis, University of Xi'an Science and Technology, 2013.

[19] J. H. Steiger, and J. M. Lind, "Statistically-Based Tests for the Number of Common Factors", Paper Presented at the Psychometric Society Meeting, Iowa City, 1980.

[20] J. H. Steiger, "Structure model evaluation and modification: An interval estimation approach", Multivariate Behavioral Research, vol. 25, pp. 173-180, 1990. 
[21] P. M. Bentler, and D. G. Bonett, "Significant tests and goodness of fit in the analysis of covariance structures", Psychological Bulletin, vol. 88, pp. 588-606, 1980.

[22] P. M. Bentler, "Comparative fit indexes in structural models", Psychological Bulletin, vol. 107, pp. 238-246, 1990.
[23] Y. J. Liu, "Study of a comprehensive assessment method for coal mine safety based on a hierarchical grey analysis", Journal of China University of Mining and Technology, vol. 17, no. 1, pp. 610, 2007.

(C) Yerong et al.; Licensee Bentham Open.

This is an open access article licensed under the terms of the (https://creativecommons.org/licenses/by/4.0/legalcode), which permits unrestricted, noncommercial use, distribution and reproduction in any medium, provided the work is properly cited. 